\title{
Nivel de formación y necesidad de capacitación en entornos virtuales como factores claves para el desarrollo académico-profesional del recurso humano en cuatro unidades académicas de la Universidad de Panamá.
}

\section{Level of formation and the need for training in virtual environments as key factors for the academic-professional development of the human resource in four academic units of the University of Panama.}

\author{
Libertad Fernández G. ${ }^{1}$, Damaris E. Rodríguez C. ${ }^{2}$, Roma Monge de Ruíz. ${ }^{3}$, Oscar E. \\ Rodríguez C. ${ }^{4}$, Raúl E. Dutari D. ${ }^{5}$
}

\begin{abstract}
${ }^{1}$ Maestría en Administración de Empresas con énfasis en Recursos Humanos; Profesora, Universidad de Panamá, Centro Regional Universitario de Veraguas; libertad.fernandez@up.ac.pa; https://orcid.org/00000002-3705-4761

${ }^{2}$ Maestría en Administración de Recursos Humanos; Universidad Especializada de las Américas, Sede de Santiago; dr090902@gmail.com; https://orcid.org/0000-0001-7225-6345

${ }^{3}$ Maestría en Administración de Empresas con énfasis en Recursos Humanos; Profesora, Universidad de Panamá, Centro Regional Universitario de Veraguas; roma.monge@up.ac.pa; https://orcid.org/0000-0002$\underline{5143-2589}$

${ }^{4}$ Maestría en Entornos Virtuales de Aprendizaje; Profesor, Universidad de Panamá, Centro Regional Universitario de Veraguas; oseroca.rodriguez@up.ac.pa; https://orcid.org/0000-0001-5438-8037

${ }^{5}$ Maestría en Computación; Profesor, Universidad de Panamá, Centro Regional Universitario de Veraguas; raul.dutari@up.ac.pa; https://orcid.org/0000-0002-7954-5999
\end{abstract}

Resumen: Con el propósito de identificar el nivel de formación y la necesidad de capacitación en entornos virtuales como factores claves para el desarrollo académico-profesional del recurso humano, se aplicó entre los meses de abril a junio del presente año, un formulario en línea de manera remota, a los coordinadores y profesores de la Facultad de Informática, Electrónica y Comunicación, en cuatro unidades académicas. El 70.73\% de estos respondieron dicho instrumento, el cual recopiló los resultados de la preparación académicaprofesional y los sistemas de gestión de aprendizaje utilizados en el desarrollo de clases no presenciales. Como aspecto concluyente, se determinó que el $41.38 \%$ cuenta con preparación académica formal a nivel de postgrado y maestría en entornos virtuales, y sólo el $20.68 \%$ utilizó la plataforma oficial del campus virtual (Educativa), durante el primer semestre del año académico en curso; lo que demanda mejores estrategias de capacitación del talento humano.

Palabras clave: recurso humano, capacitación, desarrollo académico-profesional, entornos virtuales.

Abstract: With the purpose of identifying the level of formation and the need for training in virtual environments as key factors for the academic-professional development of the human resource, an online form was applied remotely to the coordinators and professors of the Faculty of Informatics, Electronics and Communication, in four academic units between April and June of this year. $70.73 \%$ of these responded to this instrument, which compiled the results of academic-professional preparation and learning management 
systems used in the development of non-face-to-face classes. As a conclusive aspect, it was determined that $41.38 \%$ have formal academic preparation at the postgraduate and master's level in virtual environments, and only $20.68 \%$ used the official platform of the virtual campus (E-ducativa) during the first semester of the current academic year, which demands better human talent training strategies.

Key words: human resource, training, academic-professional improvement, virtual environments.

\section{Introducción}

Con el advenimiento y evolución de las llamadas Tecnologías de la Información y la Comunicación (TIC, por sus siglas), la sociedad en general ha experimentado múltiples transformaciones a lo largo del tiempo; puesto que al desarrollar muchas de las actividades consideradas como rutinarias, estas se han convertido en pilares para el avance y progreso social. Sin embargo, las condiciones que actualmente se viven como sociedad producto de la pandemia COVID-19, manifiestan sus efectos en todos los niveles sociales, institucionales y empresariales; propiciando cambios y adecuaciones en las acciones y tareas cotidianas que día a día emprendemos como entes individuales, pero a la vez generan nuevas oportunidades para reinventarnos y ser emprendedores en un mundo que ya no será igual al que se estaba acostumbrado (Escobar, 2020).

Los recientes y notables eventos sociales, las nuevas circunstancias, las normas de salud y políticas gubernamentales que inciden en el ejercicio de muchas organizaciones de nuestro país, sin importar su tamaño y actividad socio-económica, enfatizan que el capital, talento humano o capital intelectual, es el elemento clave en el desarrollo y mejora de sus operaciones como entidades económicas; por ello, es de suma importancia su cuidado y salvaguarda, ante los inminentes riesgos y amenazas que se encuentran latentes en el medio social bajo el contexto de la llamada "nueva normalidad".

Cualquiera que sea el concepto aplicado para referirse a las personas que conforman dichas organizaciones, se destacan como prioritarias e importantes para el funcionamiento y progreso de todas las entidades, ya sean públicas o privadas. Por ello, el Capital Humano se describe como los conocimientos, habilidades, capacidades y otros atributos de los empleados, que tienen valor económico para una organización (Bohlander, Snell y Morris, 2018). 
En atención a la naturaleza y actividades de las organizaciones, las condiciones actuales acentúan la utilización de un número variado de recursos y herramientas tecnológicas, para llevar a cabo muchas de las labores que hacemos a cada momento; no obstante, también es muy notable la gran dependencia que existe hacia estas, al uso constante de las redes de transmisión de datos y el acceso a internet.

Por su parte, las universidades como entidad de educación pública no escapan ante esta realidad; y se han visto obligadas a realizar múltiples tareas de adiestramiento de forma rápida en el uso de herramientas tecnológicas y sistemas de gestión de aprendizaje o de las llamadas plataformas educativas, como acciones efectivas para la preparación del estamento docente; puesto que se ha entrado al proceso denominado virtualización de la educación, que involucra un sinnúmero de cambios y transformaciones en el accionar de todas las instituciones de educación superior, y por ende de la Universidad de Panamá.

Es aquí donde la capacitación se posiciona como una estrategia y como un proceso formal inherente al desarrollo del talento humano, y se destaca como sistemática, proactiva y tecnológica; por ello, en la actualidad es una de las acciones que requiere el uso constante y dinámico de la tecnología y que su importancia y utilidad sobrepasa cualquier estimación antes hecha. Queda claro que las instituciones universitarias enfrentan uno de los mayores retos y desafíos, la promoción y transmisión del conocimiento a través de herramientas tecnológicas que apoyen el proceso de enseñar y aprender a través de los entornos virtuales.

Capacitar significa dar a los empleados nuevos o antiguos, las habilidades que requieren para desempeñar su trabajo; y que, además del análisis de las necesidades de capacitación, se identifica el entrenamiento que los individuos requieren para cubrir sus puestos de trabajo (Dessler, 2015). También destaca como un aspecto técnico, que va dirigido a la preparación en el dominio del trabajo en sí mismo, lo intelectual y lo físico; centrándose en la eficiencia, eficacia, excelencia y mejora continua (Sotomayor, 2016).

A lo largo de los años, el perfeccionamiento académico y el adiestramiento profesional, han demostrado ser acciones fundamentales en el desarrollo y evolución del talento humano en cualquier tipo de institución; en consecuencia, la capacitación es el 
proceso educativo de corto plazo, aplicado de manera metódica y organizada, por el que las personas adquieren conocimientos, desarrollan habilidades y competencias en función de objetivos definidos (Chiavenato, 2011).

Por lo antes expuesto, la capacitación como actividad de formación y mejora del talento humano en las instituciones de educación superior, cumple de forma real, efectiva y actual, su vital y muy significativa función y que, con el apoyo de herramientas tecnológicas adecuadas, se presentan como una estratégica alternativa para dar soporte al proceso de enseñanza a través de los entornos virtuales.

Es imperativo reconocer que la educación como proceso de formación, requiere de un proceso de enseñanza atractivo y de un proceso de aprendizaje productivo, que sea a su vez relevante ante la situación actual; por ello, la educación a través de los entornos virtuales es mucho más que el simple hecho de utilizar una herramienta tecnológica para impartir clases o utilizarla como recurso de enseñanza; conlleva la planificación, organización, diseño, construcción, validación formal y de la adecuación pedagógica de los materiales educativos, de la mediación didáctica en línea bajo los entornos digitales, tropicalización de los modelos pedagógicos emergentes Bravo (2020), de prácticas metodológicas cónsonas con el entorno de los discentes, de la selección real y efectiva de los recursos, del acompañamiento del tutor y de un sistema de evaluación que contemple el trabajo, compromiso, disposición de los estudiantes, de sus estilos de aprendizaje y de las facilidades de acceso a la red y conectividad (Valverde y López, 2009).

Atender las necesidades actuales de capacitación del estamento docente que forman tanto las unidades académicas objeto de estudio como el resto que conforma la Universidad de Panamá, no es una tarea fácil, ni tampoco se debe hacer de forma rápida; ya que se necesita de aplicar las mejores prácticas y técnicas de capacitación en el área de formación profesional a través de los entornos virtuales. Por ello, también es esencial analizar y aplicar algunas experiencias previas en el diseño, desarrollo y puesta en marcha de modelos de capacitación (Henríquez y otros, 2015).

Dichas necesidades toman muy en cuenta las carencias o fallas actuales que presenta un individuo en cuanto al desarrollo de las competencias o de la voluntad para realizar un 
trabajo; y a su vez, trata de como corregirlas a través de un proceso de enseñanzaaprendizaje sistemático, con objetivos muy bien definidos y evaluables (Martínez y Martínez, 2009). Por su parte, el diseño de una actividad de capacitación debe incluir múltiples elementos y aspectos, como la identificación de las necesidades emergentes, el análisis del conocimiento previo y las propias actitudes para el desempeño de las labores a desarrollar (Cejas, y otros, 2017).

Queda claro que, ejecutar una acción de capacitación trasciende el adiestramiento en una herramienta tecnológica determinada, de conocer su funcionamiento y características técnicas; requiere del análisis de los efectos de la formación en aspectos como: La Reacción de los participantes al enfrentarse a nuevas condiciones, del propio aprendizaje que incluye el desarrollo de las competencias digitales requeridas, del comportamiento y de los resultados esperados en la actividad (Dessler, 2020).

Las experiencias recopiladas durante el primer semestre del año académico en curso, son la base para la presentación de nuevas acciones en materia de capacitación del talento humano que forma parte de la Universidad de Panamá; por ello, como propósito principal del estudio, se presenta identificar el nivel de formación y la necesidad de capacitación en entornos virtuales como factores claves pare el desarrollo académico-profesional del recurso humano (personal docente) de la Facultad de Informática, Electrónica y Comunicación, en cuatro centros regionales universitarios del interior del país.

Esta es una excelente oportunidad considerando la situación actual, para destacar la gran importancia de la capacitación como estrategia de perfeccionamiento del desarrollo del talento humano, dentro de la llamada "nueva realidad".

\section{Materiales y métodos}

La estructura académica vigente en la Universidad de Panamá establece que, dentro de la Facultad de Informática, Electrónica y Comunicación (F.I.E.C, por sus siglas), existe el Departamento de Informática; y que, a su vez lo componen de cinco áreas de desarrollo de conocimiento. Una de estas es la de Informática Aplicada, Sistemas Virtuales y Multimedia, lo cual establece que son los docentes inmersos en estas los que poseen la formación 
académica y profesional para emitir juicios técnicos en materia de formación y necesidades de capacitación en entornos virtuales (Universidad de Panamá, 2020).

Se diseñó un formulario en línea y se aplicó de forma remota a toda la población de profesores especiales y regulares (41 en total), que actualmente se encuentran en la organización docente del primer semestre del año académico en curso de la FIEC, en los Centros Regionales Universitarios de Azuero, Bocas del Toro, Coclé y Las Tablas; según información recopilada a través de la comunicación vía correo electrónico con los Coordinadores de Facultad en estas sedes. (Alonso, Poveda, Pinzón, y Serrano, 2020).

Es muy importante señalar que, al hablar de población, se refiere a su totalidad, tanto de los sujetos seleccionados, como los aspectos u objetos de estudio; población es la colección completa de todos los elementos a estudiar (Del Cid, Méndez, y Sandoval, 2011). También, el concepto de población se define como la totalidad de elementos o individuos que tienen ciertas características similares y sobre las cuales se desea hacer inferencia o bien, es la unidad de análisis del estudio (Bernal, 2010).

Se destaca que, a pesar de conocer la cantidad de profesores que respondieron el formulario en línea, se realizaron los cálculos para determinar la muestra, en atención a la población señalada. La muestra es en esencia parte, un subgrupo o subconjunto de la población estudiada; a la que pertenecen ese conjunto definido como profesores al que Ilamamos población (Hernández Sampieri, y otros, 2010).

Una forma de establecer el tamaño requerido de la muestra que se debe extraer de la población de profesores a la que se le aplicaría el formulario en línea; se fundamenta en las siguientes fórmulas:

$$
\begin{aligned}
& \text { 1. } n^{\prime}=\frac{P(1-P)}{V^{2}} \\
& \text { 2. } n=\frac{n^{\prime}}{1+\frac{n^{\prime}}{N}}
\end{aligned}
$$

3. $S=s e$

Fuente: (Hernández Sampieri, Fernández Collado, y Baptista Lucio, 2010) 
Donde:

$n^{\prime}=\quad$ Tamaño de la muestra sin ajustar.

$n=\quad$ Tamaño de la muestra ajustada.

$N=\quad$ Tamaño de la población a encuestar (41 profesores).

$s e=0.06=6 \%=$ Error estándar establecido por los investigadores.

$P=0.5=50 \%=$ Probabilidad de ocurrencia de que el elemento seleccionado en la población, presente el atributo de interés en la encuesta (sin premuestreo) (Levine, y otros, 2014), (Abad y Servin, 1982).

$V^{2}=\quad$ Varianza de la población a encuestar.

$S^{2}=\quad$ Varianza de la muestra.

Sustituyendo los valores conocidos, se obtiene:

$$
\begin{aligned}
& n^{\prime}=\frac{0.5(1-0.5)}{s e^{2}}=\frac{0.25}{(0.06)^{2}}=\frac{0.25}{0.0036}=69.4444 \\
& n=\frac{69.4444}{1+\frac{69.4444}{41}}=\frac{69.4444}{1+1.6937}=\frac{69.4444}{2.6937}=25.7797 \approx 26
\end{aligned}
$$

Cabe indicar, que respondieron 29 de ellos, lo que representó un $70.73 \%$ de la población total investigada. Es decir, se deben muestrear al menos a 26 profesores de la población de 41 miembros. En consecuencia, dado que la encuesta fue respondida por 29 docentes, se puede establecer que el tamaño mínimo de la muestra es inferior a la cantidad de profesores que respondieron el formulario; significa entonces, que se trabajará con un tamaño de muestra mayor que el mínimo requerido, lo que se expresa de la siguiente manera:

$$
\begin{gathered}
\#(P T)=41, \#(T M M)=26, \#(P E)=29 \\
\therefore \\
\#(T M M) \leq \#(P E) \leq \#(P T) \wedge \forall p_{T M M} \in\{T M M\}, p_{T M M} \in\{P E\}
\end{gathered}
$$

Donde:

$P T=\quad$ Conjunto de la población total de profesores.

$T M M=\quad$ Conjunto de profesores que tiene el tamaño mínimo de muestra. 


$$
\begin{array}{ll}
P E= & \text { Conjunto de profesores encuestados. } \\
p_{T M M}=\quad & \text { Un profesor cualquiera que colaboró con la investigación, } \\
& \text { respondiendo la encuenta aplicada. }
\end{array}
$$

El instrumento en mención, contiene 11 ítems de tipo selección múltiple, el cual fue validado por tres profesores especialistas en Recursos Humanos y un profesor especialista en Entornos Virtuales de Aprendizaje, que laboran en la Universidad de Panamá, Universidad Latina y la Universidad Especializada de las Américas, todas ubicadas en el distrito de Santiago, provincia de Veraguas. Es muy importante enfatizar, que se requería del diseño de un instrumento que fuera fácil y práctico, rápido en la selección de las respuestas y que optimizara el tiempo de acceso a las redes, debido a las circunstancias actuales y que, a su vez se desconocía las condiciones de conectividad de los profesores de dicha facultad.

El formulario se dividió en dos secciones, de los cuales el $27.27 \%$ de los ítems corresponden a la primera parte de datos generales, donde se indagaron temas como: categoría del docente, años de servicios académicos, formación y títulos universitarios registrados; y el $72.73 \%$ de estos, corresponden a la segunda sección vinculada al perfeccionamiento académico-profesional en entornos virtuales, plataformas educativas seleccionadas, criterios de selección y otros elementos asociados a esta temática.

Se analizaron cinco aspectos importantes como referentes y objetos de investigación: Perfeccionamiento académico en entornos virtuales, tipo de actividad, cantidad de horas de capacitación; de igual forma, las plataformas utilizadas para desarrollar clases no presenciales bajo el entorno virtual y de los criterios técnicos-pedagógicos utilizados para dicha selección. Estos criterios sustentan la base educativa para la selección adecuada de las herramientas utilizadas como sistemas de gestión de aprendizaje (SGA, por sus siglas); puesto que desde inicios de este siglo ya se venían dando investigaciones referenciales al respecto. Tanto es así, como el estudio propuesto por Chiarani, Pianucci, y Lucero, (2004), que resalta aspectos importantes para la selección de estos recursos, destacando: Herramientas del profesor, herramientas de comunicación, herramientas del estudiante, especificaciones técnicas y herramientas de administración. Sin embargo, Salinas, (2006), 
presenta tres componentes básicos para el desarrollo y evaluación de esta actividad: componentes pedagógicos, componentes organizativos y los componentes técnicos.

Lo anterior, permite proponer un grupo de aspectos generales que servirán como referencia para la selección de una plataforma virtual que sirva de SGA, atendiendo a las condiciones y circunstancias actuales, tanto del profesor como del estudiante. Se proponen siete criterios básicos: Idioma, facilidad de descarga, costos, flexibilidad pedagógica, usabilidad (Profesores-Estudiantes), flexibilidad tecnológica y características propias de la herramienta; cada criterio debe ser evaluado por los encuestados atendiendo la siguiente escala numérica: de 1 a 7, considerando para el de mayor importancia el valor máximo de (7), y para el de menor transcendencia el valor mínimo de (1).

\section{Resultados y discusión}

La presentación de los resultados responde al análisis de los datos obtenidos en 8 de las 11 preguntas propuestas; por ello, como se observa en la figura 1, un aspecto importante es identificar la categoría de los docentes, considerando sólo a los profesores regulares y los nombrados por resolución, excluyéndose a los docentes de Banco de Datos.

Figura 1. Categoría docente, según su certificado de servicios académicos - UP.

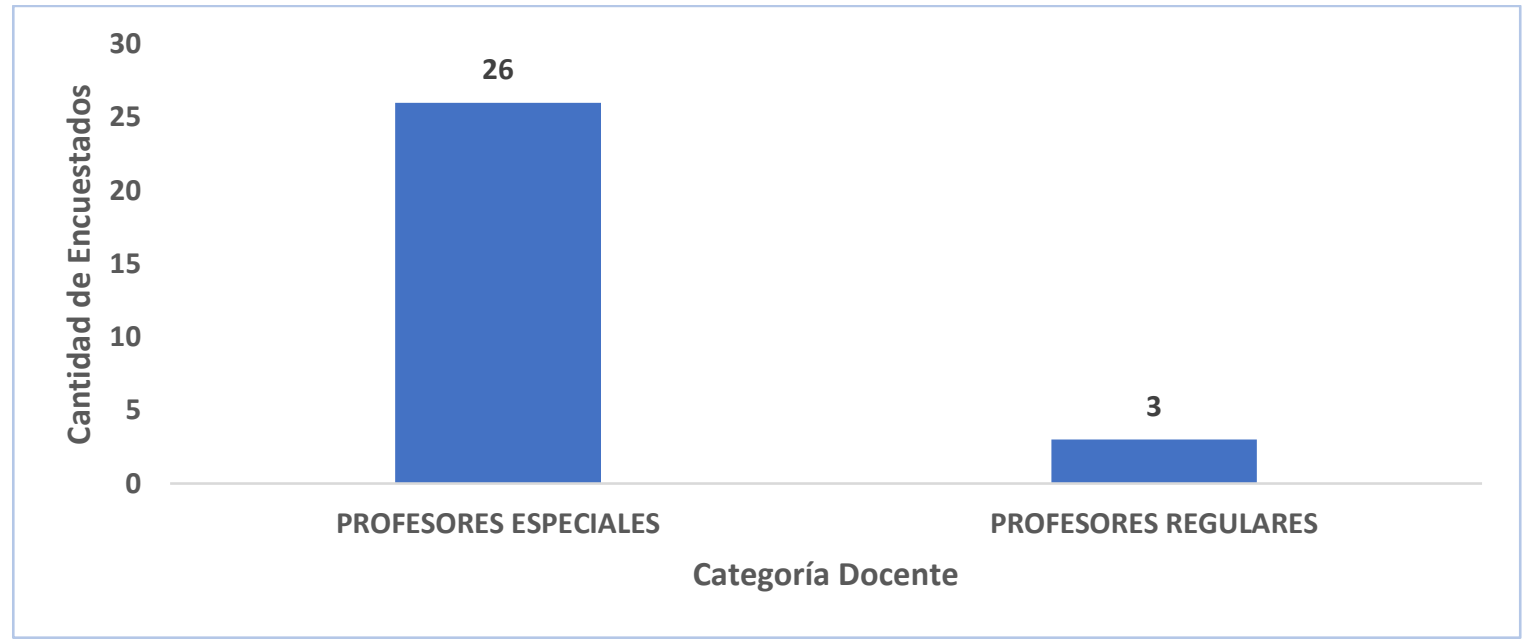

Fuente: Resultado del formulario aplicado por los autores. 
Según su categoría, 26 (89.65\%) de los docentes encuestados pertenecen al grupo de profesores especiales nombrados por resolución y el sólo $3(10.35 \%)$ corresponden a profesores regulares.

Muy asociado a la pregunta anterior, se indagó sobre los años de servicios académicos dentro de la Universidad de Panamá, lo cual se presenta en la figura 2.

Figura 2. Años de servicios académicos - UP.

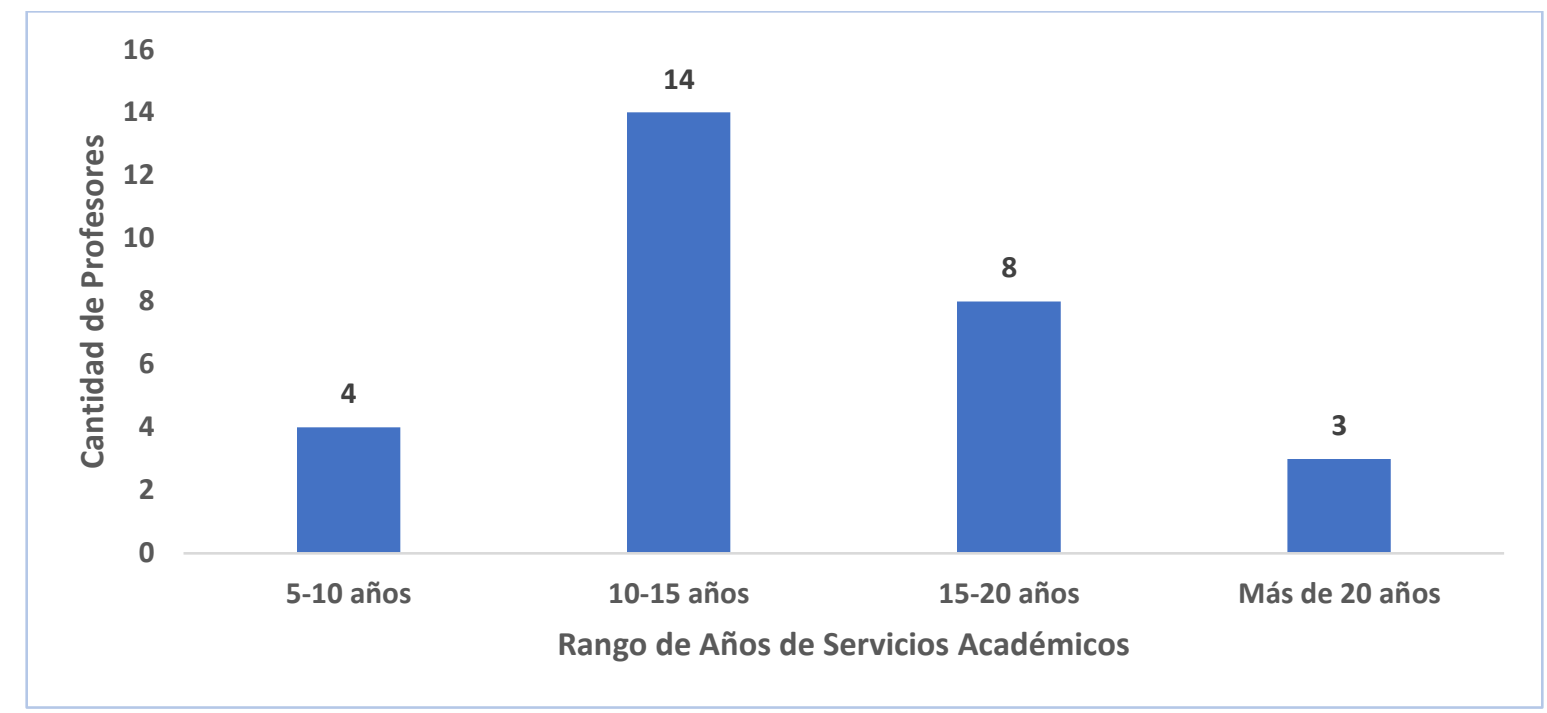

Fuente: Resultado del formulario aplicado por los autores.

Considerando los años de servicios académicos, los 29 (100\%) de los encuestados tienen nombramiento por resolución y cuentan con un promedio superior a los 10 años de servicios en esta institución. Lo anterior pone de manifiesto que un alto porcentaje de estos posee mayor experiencia, conoce la estructura organizativa y funcional, la normativa y la cultura organizacional de la Universidad de Panamá, lo que coadyuva a enfrentar con mayor facilidad las exigencias profesionales ante la situación actual.

La figura 3 muestra la formación académica-profesional de los profesores encuestados. 
Figura 3. Grado académico / título universitario

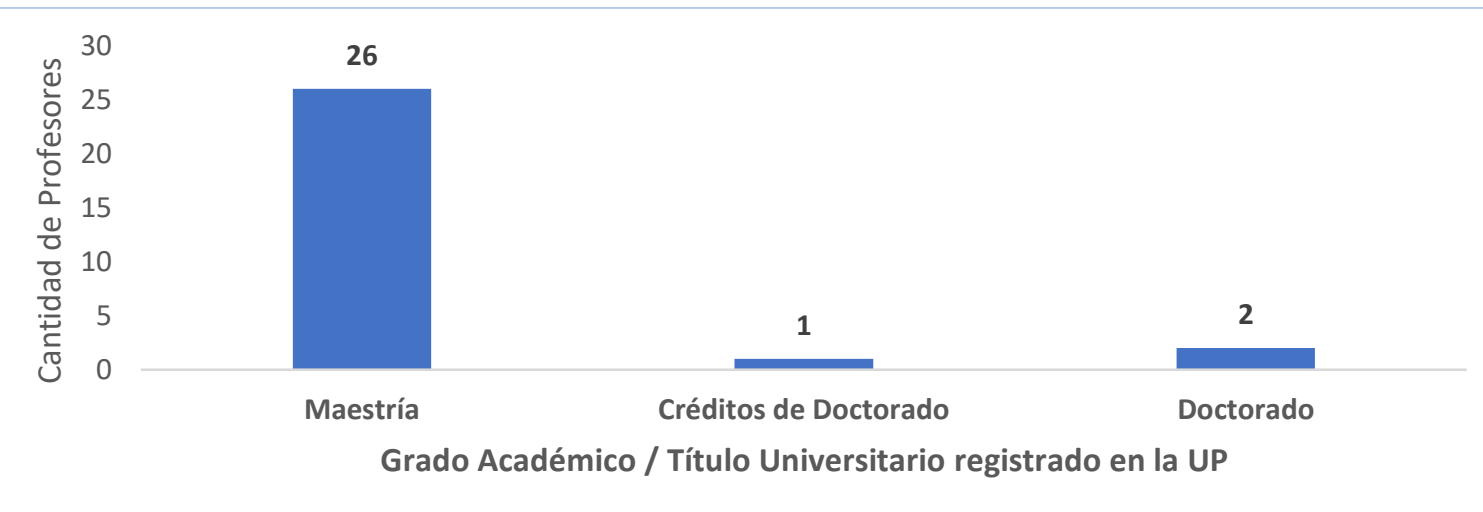

Fuente: Resultado del formulario aplicado por los autores.

Es muy importante recalcar que 26 (89.65\%), posee el grado de Maestría y 3 (10.35\%) tiene formación a nivel de doctorado. Lo anterior evidencia que existe una relación directa en muchas de las características de los encuestados, ya que se puede inferir que 22 (75.86\%) tienen entre 10 a 20 años de servicios académicos y, el 100\% de estos posee como mínimo el grado profesional de maestría; lo cual es un requerimiento para ambas categorías de docentes (Universidad de Panamá, Acuerdo №5-10 de 17 de junio de 2010).

Con relación al perfeccionamiento académico como parte de la formación profesional en entornos virtuales, la figura 4 muestra los siguientes hallazgos.

Figura 4. Perfeccionamiento académico como parte de su formación profesional.

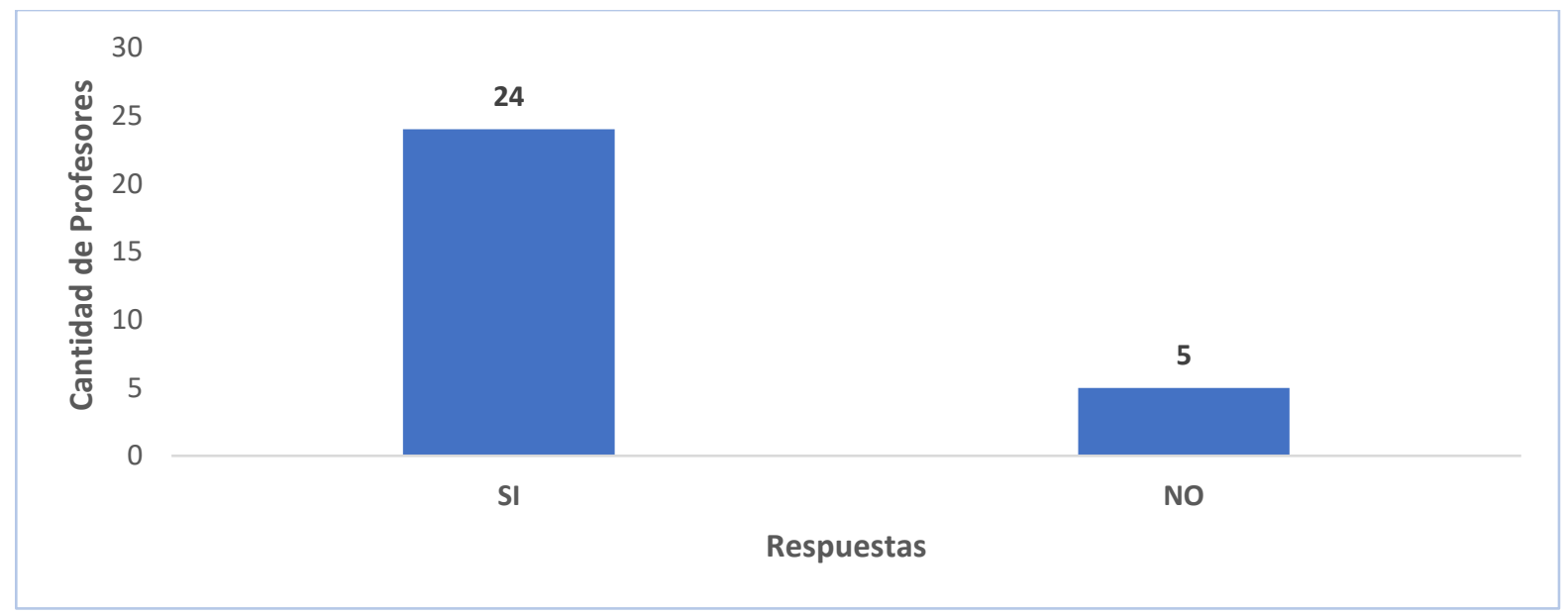

Fuente: Resultado del formulario aplicado por los autores. 
Un aspecto interesante que se presenta en los resultados obtenidos es que sólo 5 (17.24\%) de los encuestados respondieron que no han recibido capacitación en el área de entornos virtuales, lo que destaca como muy buen resultado. Sin embargo, al seguir investigando sobre las actividades de perfeccionamiento profesional en las que han participado dichos docentes, se observó que 12 (41.37\%) de estos posee formación académica de postgrado y maestría, tal como se muestra en la figura 5; lo que denota que menos de la mitad cuentan con la preparación formal requerida para enfrentar el desafío de las clases no presenciales.

Es muy importante señalar, que la formación de postgrado tiene una duración promedio de 10 a 12 meses; y a su vez, la maestría transciende los 24 meses de formación; lo que permite adquirir las competencias para atender la modalidad de la educación a través de los entornos virtuales.

Figura 5. Actividad de perfeccionamiento académico-profesional.

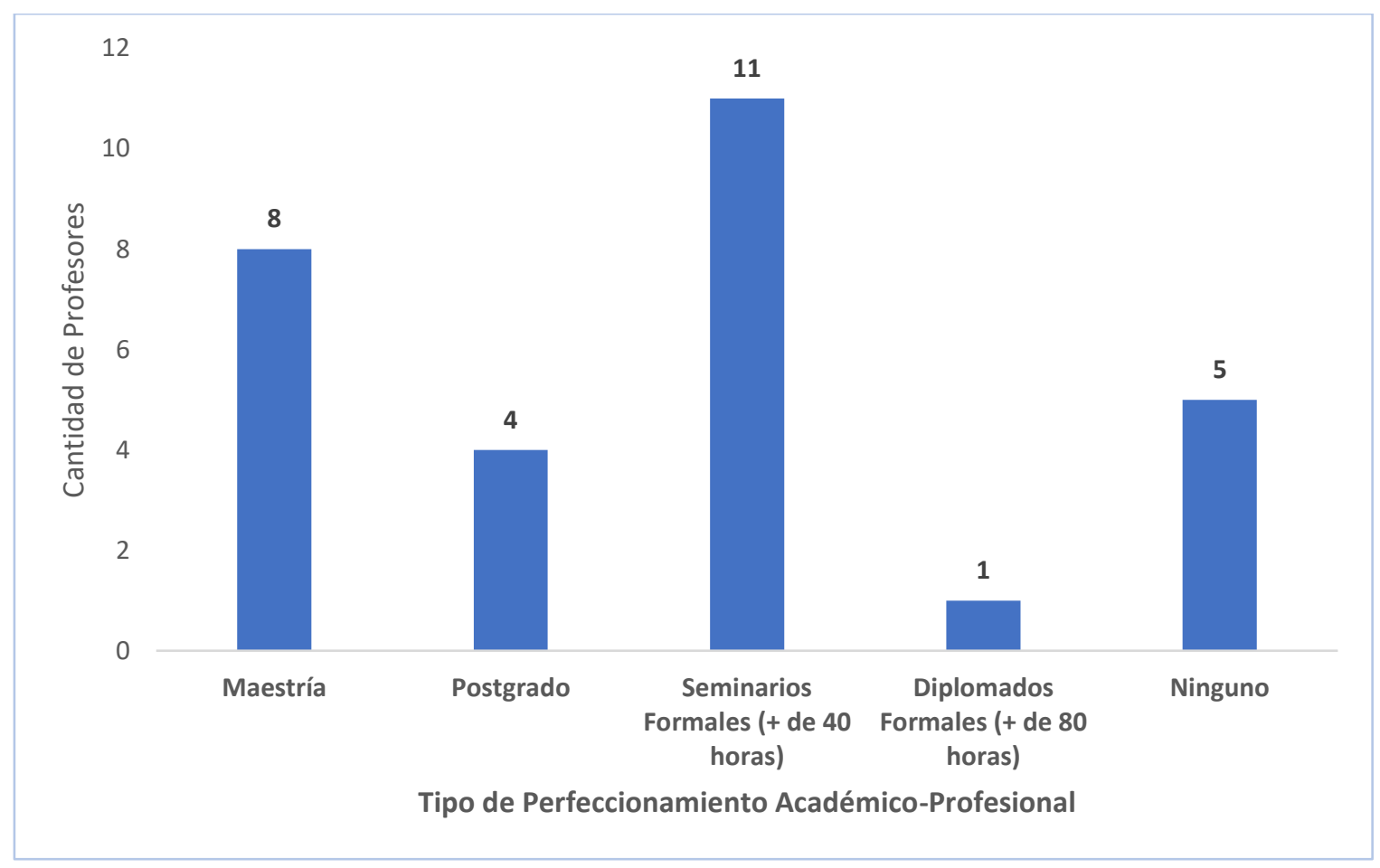

Fuente: Resultado del formulario aplicado por los autores. 
Con relación a la actividad de seminarios de más de 40 y 80 horas de capacitación, 12 $(41.37 \%)$ ha recibido alguna formación en esta modalidad; y a su vez, un aspecto relevante es que sólo 5 (17.24\%) de los encuestados respondieron que no han recibido capacitación en entornos virtuales, lo que permite inferir que 17 (58.62\%) corresponde a un grupo mayoritario sólo ha obtenido algún tipo de perfeccionamiento académico en esa área de conocimiento, lo cual ya es una cifra preocupante y encendería las alarmas de la pronta necesidad de capacitación, no sólo en el uso de una herramienta tecnológica, sino de la formación profesional en entornos virtuales que apoyen el proceso de enseñar y aprender.

Un aspecto que corrobora la importancia de la investigación, la correspondencia a las condiciones actuales y que trasciende las fronteras nacionales, es el estudio desarrollado por Valdés (2011), el cual destaca la alta necesidad de capacitación en la formación pedagógica en el uso de las tecnologías de la información y comunicación en los docentes. También se destaca el estudio realizado por Parra y Rodríguez (2016), que enfatiza el proceso de capacitación como elemento importante y base para la gestión de la calidad dentro de las organizaciones.

Las incertidumbres que surgieron al inicio del semestre del presente año académico, dieron paso a la selección de un sinnúmero de herramientas tecnológicas que servirían en teoría, para desarrollar la acción docente a través de las clases no presenciales; sin embargo, a pesar de las acciones de capacitación aceleradas y otros esfuerzos que se promocionaron para el uso de ciertas plataformas, de los acuerdos y convenios institucionales con empresas proveedoras del acceso a las redes de transmisión de datos (no requerían consumo de datos a los estudiantes), se indagó sobre las plataformas utilizas para desarrollar la praxis docente y los resultados se presentan en la figura 6. 
Figura 6. Plataforma educativa que utiliza como herramienta principal para impartir clases no presenciales.

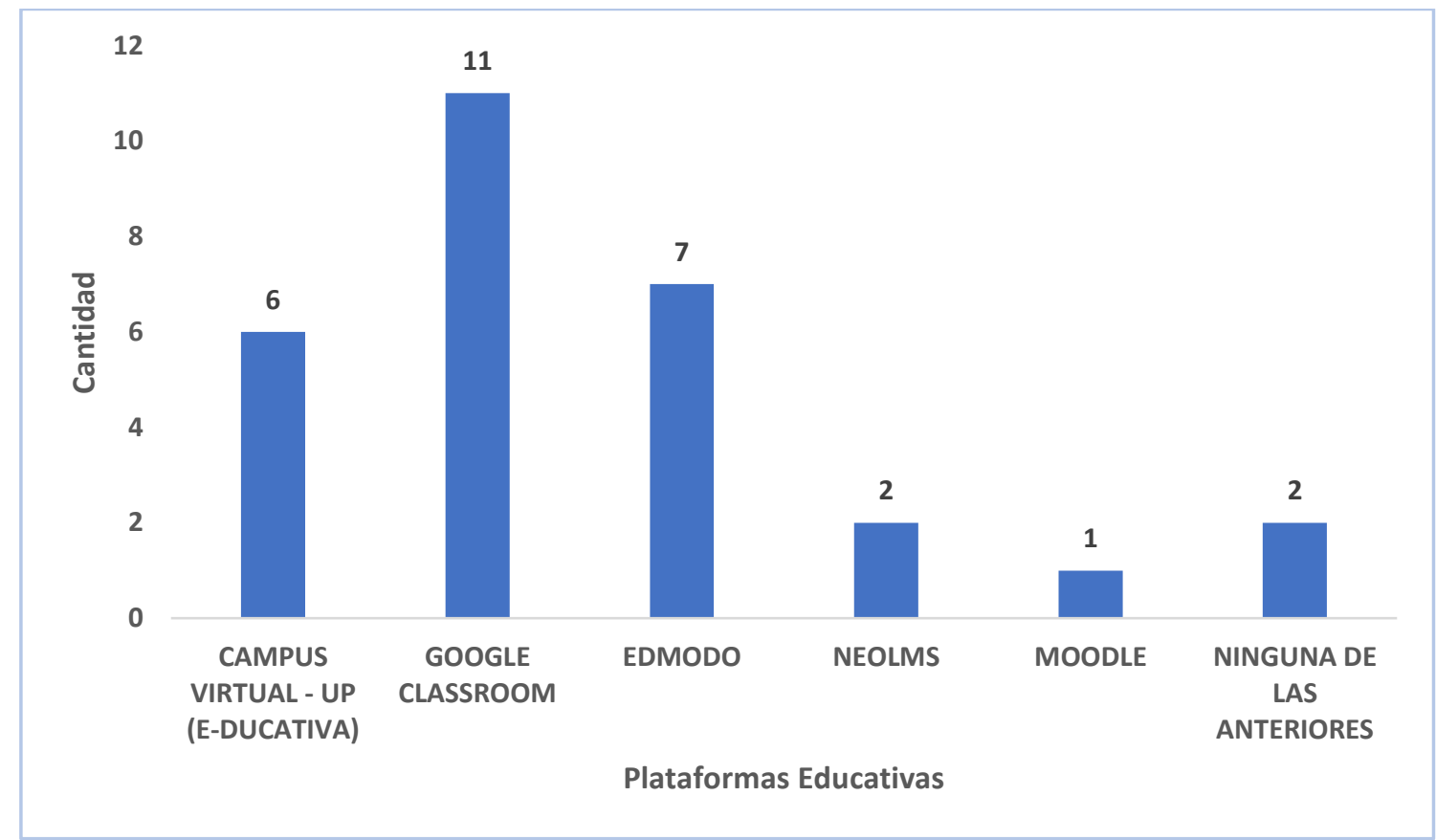

Fuente: Resultado del formulario aplicado por los autores.

De los encuestados, 11 (37.93\%) respondió que utiliza Google Classroom como plataforma educativa durante el primer semestre del año académico en curso, 7 (24.13\%) utiliza Edmodo como recurso educativo y sólo 6 (20.68\%) recurre el uso de una de las plataformas que posee la Universidad de Panamá.

Los resultados evidencian, el poco uso de las plataformas sugeridas por la Institución (E-ducativa y UPVirtual), lo que implica que la implementación para el uso de estas, demanda el estudio de las necesidades de capacitación para su utilización; ya que la herramienta con mayor porcentaje no figura entre las propuestas en la Reunión Ampliada No.3-20, celebrada el 11 de marzo de 2020 del Consejo Académico de la Universidad de Panamá (Universidad de Panamá, 2020).

Un aspecto muy interesante que se asocia a la temática anterior es el desarrollo de competencias digitales en el estamento docente, como lo propuesto por Rodríguez (2016), en donde plasma la caracterización de la necesidad de apoyo técnico y de la capacitación de docentes universitarios en el manejo de herramientas como Moodle. 
En otro orden de ideas, para comprender los resultados de la figura 7, se explica un grupo de criterios y sus referencias técnicas para la selección objetiva y profesional de un determinado recurso tecnológico; por ello, en la tabla 1, se presenta la definición de estos, los cuales fueron diseñados por los autores y se derivan de los aspectos y componentes señalados previamente por otros investigadores.

Tabla 1. Criterios propuestos para la selección de un SGA.

\begin{tabular}{|c|c|c|c|c|c|c|}
\hline IDIOMA (I) & $\begin{array}{l}\text { FACILIDAD } \\
\text { DE } \\
\text { DESCARGA } \\
\text { (FD) }\end{array}$ & $\begin{array}{c}\text { COSTOS } \\
\text { (Co) }\end{array}$ & $\begin{array}{l}\text { FLEXIBILIDAD } \\
\text { PEDAGÓGICA } \\
\text { (FP) }\end{array}$ & $\begin{array}{l}\text { USABILIDAD } \\
\text { (PROFESORES- } \\
\text { ESTUDIANTES) } \\
\text { (U) }\end{array}$ & $\begin{array}{l}\text { FLEXIBILIDAD } \\
\text { TECNOLÓGICA } \\
\text { (FT) }\end{array}$ & $\begin{array}{l}\text { CARACTERÍSTICAS } \\
\text { PROPIAS DE LA } \\
\text { HERRAMIENTA } \\
\text { (CPH) }\end{array}$ \\
\hline $\begin{array}{l}\text { Conjunto de } \\
\text { signos } \\
\text { tradicionales } \\
\text { utilizados } \\
\text { por una } \\
\text { comunidad } \\
\text { de personas } \\
\text { para } \\
\text { comunicarse } \\
\text { de forma } \\
\text { oral o por } \\
\text { escrito. }\end{array}$ & $\begin{array}{l}\text { Forma } \\
\text { simple de } \\
\text { obtener } \\
\text { información, } \\
\text { contenidos } \\
\text { y recursos a } \\
\text { través de } \\
\text { una } \\
\text { conexión a } \\
\text { internet. }\end{array}$ & $\begin{array}{l}\text { Cantidad } \\
\text { de dinero } \\
\text { que cuesta } \\
\text { o tiene } \\
\text { valor } \\
\text { monetario } \\
\text { una cosa u } \\
\text { objeto. }\end{array}$ & $\begin{array}{l}\text { Capacidad y } \\
\text { Facilidad de } \\
\text { adaptación } \\
\text { de una } \\
\text { herramienta } \\
\text { o recurso al } \\
\text { proceso de } \\
\text { enseñanza y } \\
\text { aprendizaje. }\end{array}$ & $\begin{array}{l}\text { Atributos, } \\
\text { elementos y } \\
\text { componentes } \\
\text { que facilita el } \\
\text { uso de una } \\
\text { cosa y } \\
\text { objeto. }\end{array}$ & $\begin{array}{l}\text { Capacidad y } \\
\text { Facilidad de } \\
\text { adaptación e } \\
\text { integración } \\
\text { de una } \\
\text { herramientas } \\
\text { y recurso, } \\
\text { con otros } \\
\text { diferentes. }\end{array}$ & $\begin{array}{l}\text { Elementos } \\
\text { propios y } \\
\text { particulares que } \\
\text { distinguen una } \\
\text { herramienta } \\
\text { tecnológica. }\end{array}$ \\
\hline
\end{tabular}

Fuente: Elaborado por los autores.

Lo antes expuesto permite expresar en la figura 7, que 21 (72.41\%) de los encuestados, seleccionaron el criterio de Idioma, como un factor fundamental para la selección de un SGA; sin embargo, algo muy interesante es que 4 de los criterios aplicados (facilidad de descarga, flexibilidad pedagógica, usabilidad y características propias de la herramienta) obtuvieron el mismo valor de 14 (48.27\%), muy seguido de los costos 13 (44.82\%) y finalmente flexibilidad tecnológica 12 (41.37\%). 
Figura 7. Criterios propuestos para la selección de una plataforma virtual educativa.

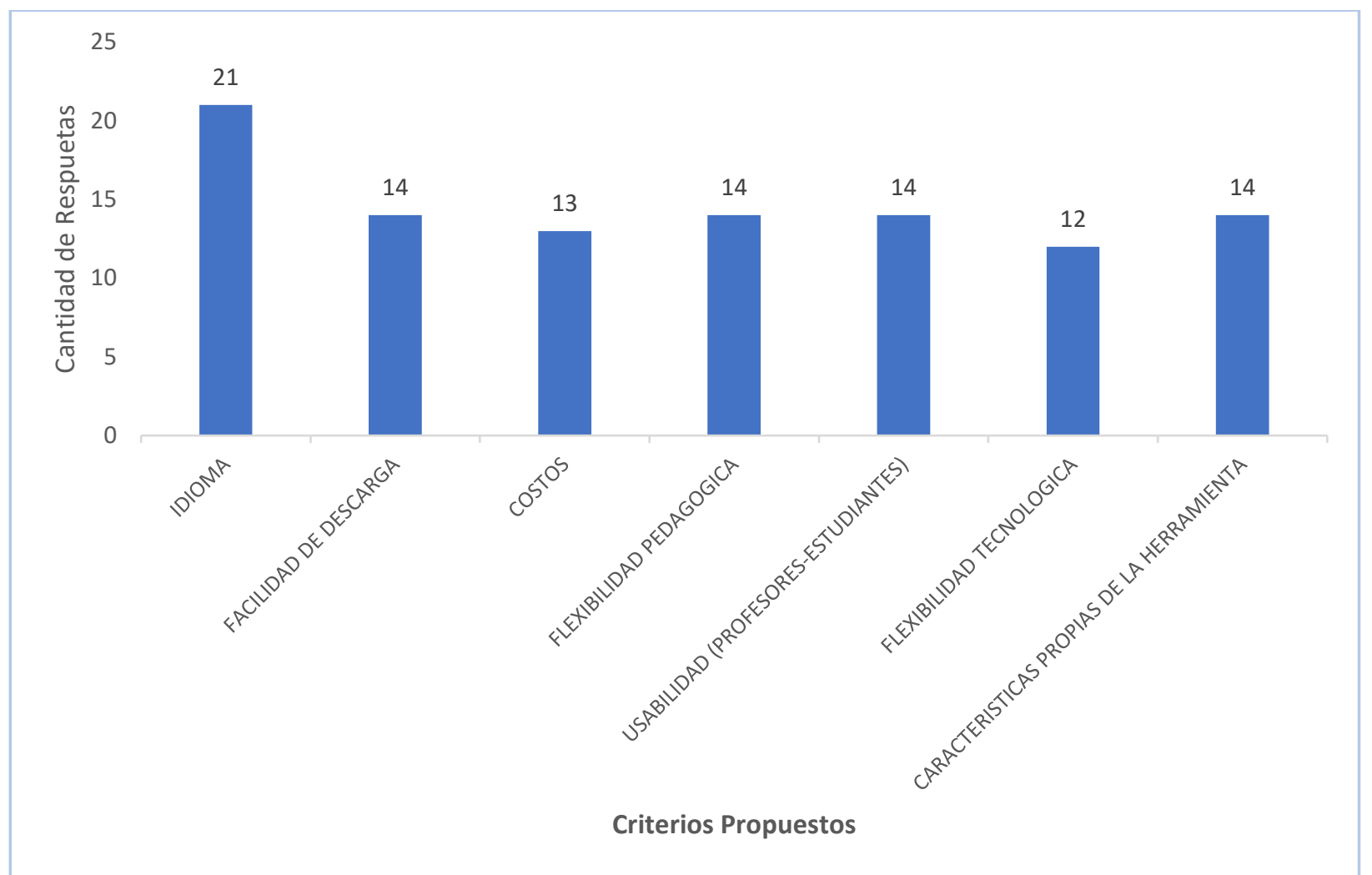

Fuente: Resultado del formulario aplicado por los autores.

Cada encuestado seleccionaba un criterio y le asignaba un valor establecido en el rango de 1 a 7; lo cual permitía que se identificara un índice del criterio, atendiendo a la mayor cantidad de repeticiones obtenidas como se puede observar en la figura 8; por consiguiente:

$$
\forall I C_{j} \in N, 1 \leq j \leq 7, \forall k \in N, 1 \leq k \leq C D E \quad \therefore \quad I C_{j}=\frac{\sum_{k=1}^{C D E} C_{j, k}}{C D E}
$$

Donde:

$j=\quad$ Cantidad de criterios propuestos por los autores (7)

$I C_{j}=\quad$ Índice calculado para el $j$ - ésimo criterio, $0 \leq I C_{j} \leq 1$

$C D E=\quad$ Cantidad de docentes encuestados

$C_{j, k}=\quad$ Valor asignado por el $k$-ésimo docente encuestado, al $j$-ésimo criterio 
Figura 8. Índice del Criterio.

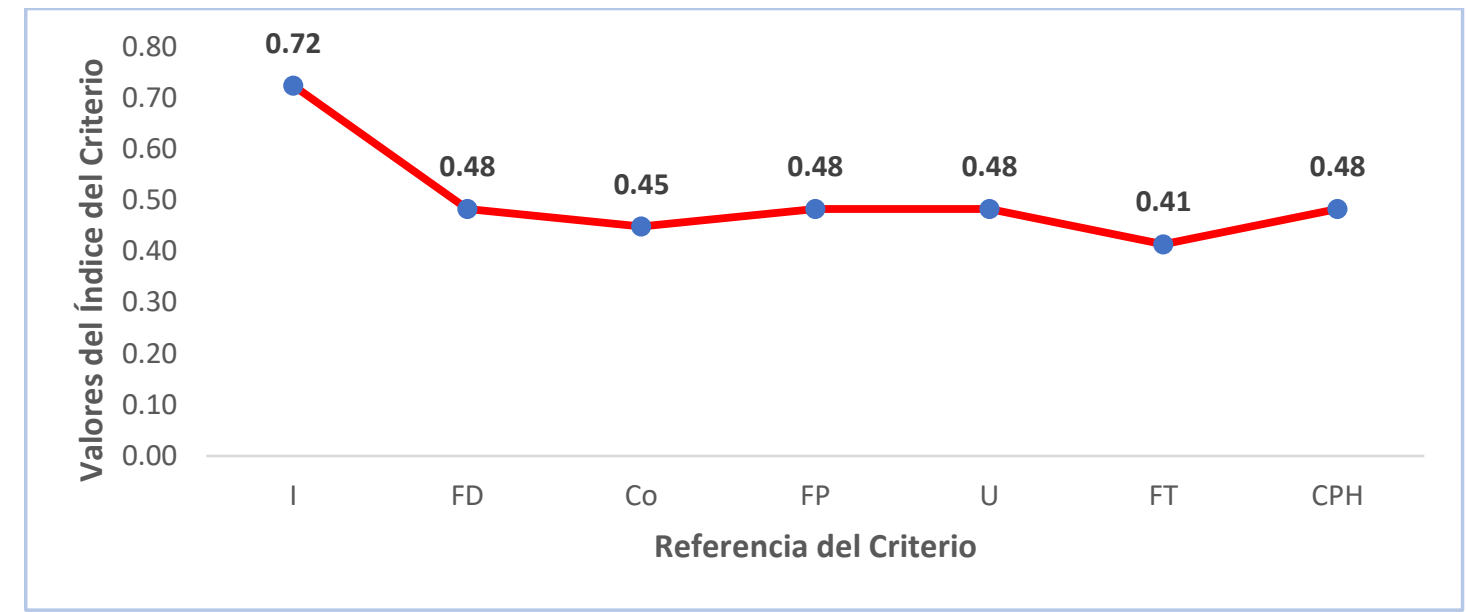

Fuente: Elaborado por los autores.

Cada resultado de la figura 8 muestra las evidencias de los criterios con mayor ponderación seleccionados por los encuestados, atendiendo a sus conocimientos y punto de vista técnico-académico.

Lo anterior fija las bases para determinar científicamente a través de la aplicación de un instrumento, los aspectos más o menos sobresalientes que deben ser considerados a la hora de diseñar y elaborar un plan de capacitación profesional; que cumpla con las necesidades y carencias detectadas, a fin de coadyuvar al desarrollo académico y profesional del talento humano que labora en la institución.

Finalmente, también se investigó sobre la herramienta de comunicación que fue utilizada como medio principal para establecer el enlace educativo con los estudiantes, lo que se presenta de manera gráfica en la figura 9. 
Figura 9. Herramienta utilizada como medio principal de comunicación.

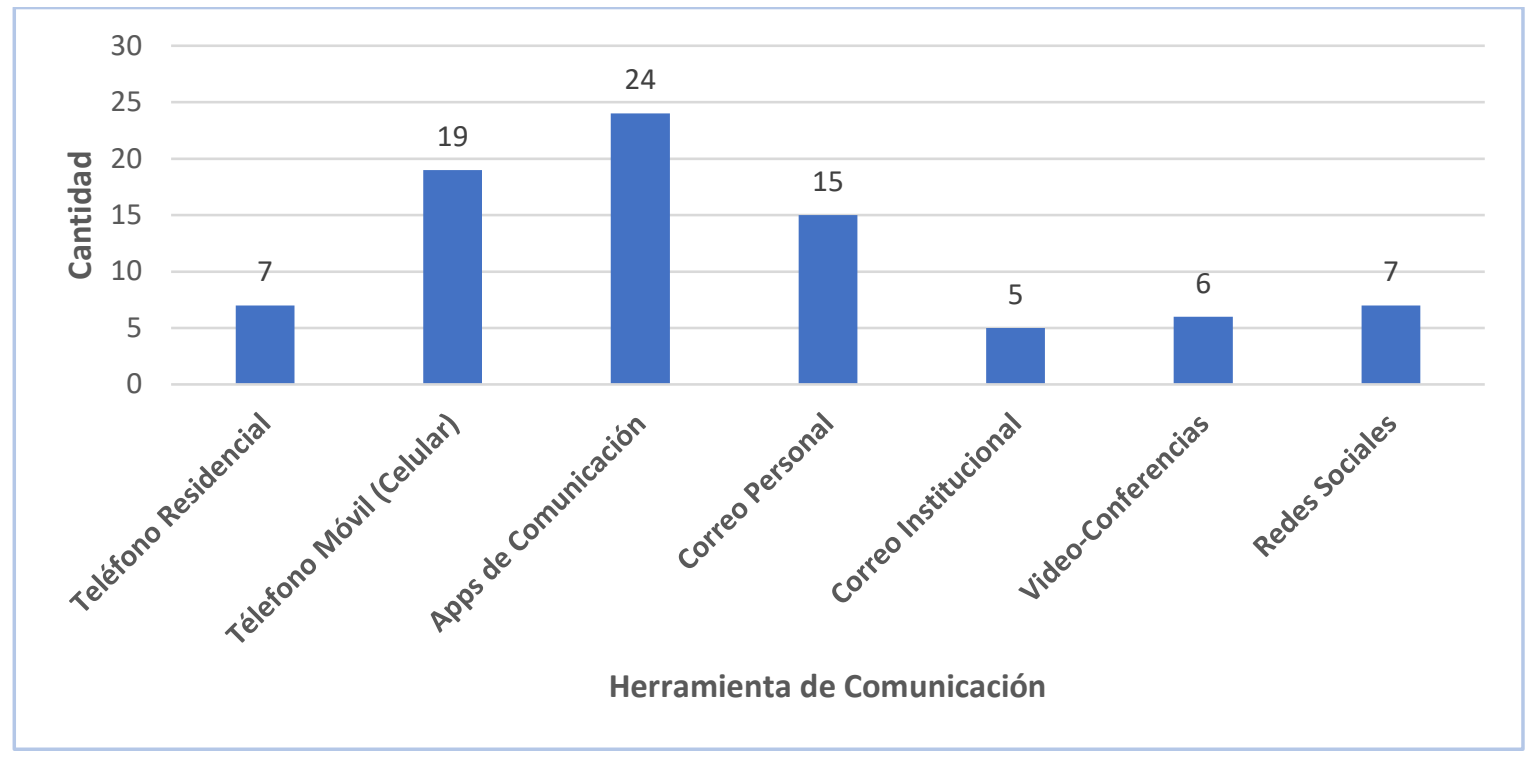

Fuente: Resultado del formulario aplicado por los autores.

La herramienta de comunicación que mayormente fue utilizada es el Apps de comunicación, que representa 24 (82.75\%), seguidamente está el teléfono móvil /celular con 19 (65.51\%) y el correo personal con 15 (51.72\%). Sin embargo, estos medios no son los que oficialmente se presentan como recursos que la institución brinda tanto a los docentes como a los estudiantes. Por lo anterior, un aspecto muy importante que surge de estos resultados es que sólo 5 (17.24\%) de los encuestados, utilizó el correo institucional como medio de comunicación con sus estudiantes, lo que se asocia a los resultados señalados en la figura 6, y que evidencia el poco uso de las plataformas educativas sugeridas por la Universidad de Panamá.

Por lo antes expuesto, se han presentado los argumentos y resultados obtenidos a través del formulario en línea de forma remota aplicado a los profesores de las cuatro unidades académicas antes señaladas.

\section{Conclusiones}

- Las condiciones imperantes al iniciar el primer semestre del año académico 2020 demandaron el uso de la virtualidad como alternativa para los procesos de enseñanza y aprendizaje. No obstante, se contó con escasas o mínimas orientaciones para 
enfrentar la situación actual, lo que provocó que el estamento docente utilizara las herramientas tecnológicas que mayor facilidad le ofrecería para el desarrollo de las clases no presenciales, dificultando la uniformidad en el uso de las mismas, con grandes perjuicios para los usuarios (profesores y estudiantes), al requerir el manejo de diversas plataformas.

- Si se contrasta el tipo de perfeccionamiento académico-profesional, se acentúa los que no han participado y los que tienen capacitación en entornos virtuales suman 17 (58.61\%) de los encuestados, sólo han participado como máximo en diplomados de 80 horas; sin embargo, sólo 12 (41.39\%) obtienen estudios formales a nivel de postgrado y maestría.

- Un aspecto muy importante relacionado a la selección de sistemas de gestión de aprendizaje (plataformas educativas) como soporte al proceso de enseñanza y aprendizaje, destaca que 23 (79.31\%) de los encuestados utilizó otros SGA diferentes, a las recomendadas por la Universidad de Panamá a través del acuerdo de la reunión ampliada No.3-20, celebrada el 11 de marzo de 2020 en el Consejo Académico.

- De lo anterior se puede inferir varias situaciones vinculadas al estudio, las cuales se centran en el desconocimiento del acuerdo académico, en cuanto al uso de las plataformas sugeridas, carencias en las competencias digitales y pedagógicas para su utilización y de la falta de capacitación y formación académico-profesional en este aspecto.

- Si analizamos las herramientas que han tenido menor relevancia para enfrentar este nuevo panorama, se presenta que tanto las plataformas como el correo institucional (recomendadas por la universidad), fueron las menos utilizadas por los docentes; a pesar de que ambas herramientas facilitan considerablemente el proceso de matrícula de los estudiantes y la propia gestión educativa de los cursos.

- Al utilizar un Índice de Criterio como aspecto técnico, se establece una relación numérica entre un ítem con mayor nivel de respuestas versus una determinada cantidad de participaciones; lo cual permitiría establecer científicamente la correspondencia de los elementos o aspectos que deben ser atendidos como carencias 
o fallas para diseñar y ejecutar un plan de capacitación real y efectivo dentro de la institución.

- La disrupción de las actividades educativas a nivel superior, es la mejor oportunidad para planificar, organizar, diseñar, elaborar, ejecutar y evaluar los planes de capacitación que actualmente se desarrollan dentro de la Universidad de Panamá; especialmente si se considera que un aspecto es adiestrar en el uso de una determinada herramienta tecnológica (plataforma educativa) y otra cosa muy distinta es conocer los principios, fundamentos de la educación y formación en entornos virtuales, como una actividad de perfeccionamiento académico-profesional.

- Se debe retomar acciones administrativas, a fin de promover y dar reapertura al programa de postgrado, maestría y hasta doctorado en entornos virtuales de aprendizaje ofrecidos en años atrás en la Universidad de Panamá.

- Para finalizar, es justo y necesario que la Universidad de Panamá como la primera casa de estudios de educación superior, se haga sentir a través de la puesta en marcha de nuevas estrategias educativas al adecuar sus ofertas académicas a los cambios que la sociedad requiere con extrema urgencia.

\section{Agradecimientos}

A los Coordinadores y Profesores de la Facultad de Informática, Electrónica y Comunicación de los Centros Regionales Universitarios de Azuero (Luis Carlos Poveda), Bocas del Toro (Daniel Serrano), Coclé (Doris Pinzón) y Los Santos (Germán Alonso), que respondieron el formulario en línea y brindaron su tiempo en la consecución de la información solicitada.

\section{Referencias bibliográficas}

Abad, A., Servin, L. A. (1982). Introducción al muestreo (Segunda ed.). México, D. F., México: LIMUSA.

Bernal, C. A. (2010). Metodología de la investigación (Tercera ed.). Bogotá, Colombia: Prentice Hall. 
Bohlander, G., Snell, S., y Morris, S. (2018). Administración de recursos humanos (Decimoséptima ed.). México, México: Cengage Learning Editores S.A.

Bravo, E. A. (2020). Secuencia operativa para la mediación didáctica en línea. Mediación didáctica (UP Virtual). Panamá: UP - Dirección de Tecnología Educativa.

Cejas, M., Vásquez, G., Chirinos, N., Hernández, G., Sandoval, L., Lozada, B., y Anzola, A. (2017). Administración de recursos humanos: La Arquitectura estratégica de las Organizaciones. Ecuador: Universidad de las Fuerzas Armadas (ESPE).

Chiarani, M., Pianucci, I., Lucero, M. (2004). Criterios de evaluación de plataformas virtuales de código abierto para ambientes de aprendizajes colaborativos. VI Workshop de Investigadores en Ciencias de la Computación. Neuquén - Argentina.

Chiavenato, I. (2011). Administración de recursos humanos (Novena ed.). México, D.F., México: McGraw-Hill.

Del Cid, A., Méndez, R., Sandoval, F. (2011). Investigación: fundamentos y metodología (Segunda ed.). Naucalpan de Juárez, México: Prentice Hall.

Dessler, G. (2015). Administración de recursos humanos (Decimocuarta ed.). México, México: Pearson.

Dessler, G. (2020). Human resource management (Decimosexta ed.). United States: Pearson.

Escobar, C. (Julio de 2020). Las transformaciones sociales en la vida cotidiana que trae consigo la pandemia. Asociación de Universidades (Grupo Montevideo). Recuperado de: http://grupomontevideo.org/sitio/noticias/las-transformaciones-sociales-en-lavida-cotidiana-que-trae-consigo-la-pandemia/

Henríquez G., G., Veracoechea F., B., Papale C., J. F., Berrios R., A. T. (2015). Modelo de capacitación docente para entornos virtuales de aprendizaje (caso decanato de ciencias de la salud de la UCLA). RIED: Revista Iberoamericana de Educación a Distancia (UNED), 18 (1), 67-90. Recuperado de http://espacio.uned.es/fez/view/bibliuned:revistaRied-2015-18-1-7030

Hernández S. R., Fernández C. C., Baptista L. P. (2010). Metodología de la investigación (Quinta ed.). México D.F., México: McGraw-Hill. 
Levine, D. M., Krehbiel, T. C., Berenson, M. L. (2014). Estadística para administración (Sexta ed.). Naucalpan de Juárez, México: Pearson Educación.

Martínez E., E., Martínez A., F. (2009). Capacitación por competencias (Principios y Métodos). Santiago de Chile, Chile: S/E.

Parra P., C., Rodríguez F., F. (2016). La capacitación y su efecto en la calidad dentro de las empresas. Universidad de Guadalajara (UDGVirtual). Recuperado de http://biblioteca.udgvirtual.udg.mx/ispui/handle/123456789/3192

Rodríguez E., H. (2016). Desarrollo de habilidades digitales docentes para implementar ambientes virtuales de aprendizaje en la docencia universitaria. Revista de investigaciones en educación (इ0ФIA-SOPHIA). ISSN 23460806, 12(2), 261-270. Recuperado de http://contexto.ugca.edu.co/index.php/sophia/article/view/561

Salinas, J. (2006). Herramientas para la formación del profesorado. Congreso Internacional EDUTEC 2005. Formación del profesorado y Nuevas Tecnologías. Santo Domingo.

Sotomayor, A. A. (2016). Administración de recursos humanos. Monterrey, México: Universitaria UANL.

Universidad de Panamá. (11 de marzo de 2020). Universidad de Panamá (Consejo Académico). Recuperado de https://secretariageneral.up.ac.pa/sites/secretariageneral/files/2020-

\section{5/C Acad N\%C2\%B03-20.pdf}

Universidad de Panamá. (15 de julio de 2020). Universidad de Panamá (Vicerrectoría Académica). Recuperado de https://viceacademica.up.ac.pa/sites/viceacademica/files/201904/Estructuras\%20Acad\%C3\%A9micas.pdf

Universidad de Panamá, (17 de junio de 2010). Universidad de Panamá (Vicerrectoría Académica). Reglamento para el nombramiento por resolución de profesores especiales y asistentes (Acuerdo №5-10 de 17 de junio de 2010).

Valdés C., A. A., Angulo A., J., Urías M., M. L., García L., R. I., y Mortis L., S. V. (2011). Necesidades de capacitación de docentes de educación básica en el uso de las TIC. 
101. Visión Antataura, Vol.4, No.2, Diciembre 2020 - Mayo 2021

Depósito de Investigación Universidad de Sevilla (idUS). Recuperado de https://idus.us.es/handle/11441/45678

Valverde B., J., y López M., E. (2009). Modelos pedagógicos en la docencia universitaria a través de entornos virtuales de enseñanza-aprendizaje. Campo Abierto, 28(2), 47-68. Recuperado de https://mascvuex.unex.es/revistas/index.php/campoabierto/article/view/1957 